\title{
Nicolas Bernard, Géographie du nautisme
}

Coll. "Espace et territoires", Presses universitaires de Rennes, 2016

\section{Anne Gaugue}

\section{OpenEdition}

\section{Journals}

Édition électronique

URL : http://journals.openedition.org/tourisme/1333

DOI : 10.4000/tourisme. 1333

ISSN : 2492-7503

Éditeur

Éditions touristiques européennes

\section{Référence électronique}

Anne Gaugue, « Nicolas Bernard, Géographie du nautisme », Mondes du Tourisme [En ligne], 12 | 2016, mis en ligne le 01 décembre 2016, consulté le 25 septembre 2020. URL : http://

journals.openedition.org/tourisme/1333; DOI : https://doi.org/10.4000/tourisme.1333

Ce document a été généré automatiquement le 25 septembre 2020.

\section{cc)}

Mondes du tourisme est mis à disposition selon les termes de la licence Creative Commons Attribution - Pas d'Utilisation Commerciale - Pas de Modification 4.0 International. 


\title{
Nicolas Bernard, Géographie du
}

\section{nautisme}

Coll. "Espace et territoires”, Presses universitaires de Rennes, 2016

\author{
Anne Gaugue
}

\section{RÉFÉRENCE}

Nicolas Bernard, Géographie du nautisme, « Espace et territoires », Presses universitaires de Rennes, 2016.

1 C'est à une réflexion approfondie sur la notion de territoire que Nicolas Bernard nous convie à partir de l'exemple du nautisme, considéré comme une activité économique et une pratique récréative. En s'attachant à la dimension spatiale des activités nautiques voile, surf, kite-surf, canoë-kayak, etc. - qu'elles soient sportives, ludiques ou touristiques, Nicolas Bernard révèle un système complexe de territorialités.

2 L'ouvrage comporte quatre parties : la première aborde l'évolution du nautisme comme pratique de loisirs ainsi que la place accordée au nautisme dans la recherche en sciences humaines et sociales. Dans la seconde partie, l'ancrage territorial du nautisme est analysé à partir de trois approches territoriales: conceptuelle, idéelle et fonctionnelle. L'impact du nautisme sur le développement local et l'économie touristique fait l'objet de la troisième partie et la dernière partie est consacrée aux potentialités du nautisme, considéré tout à la fois comme une ressource territoriale à optimiser et un champ de recherche offrant des perspectives scientifiques à explorer. Pour étayer sa démonstration, Nicolas Bernard s'appuie sur des exemples choisis sur l'ensemble du littoral français et des voies d'eaux intérieures.

3 Cet ouvrage démontre de façon magistrale et convaincante comment se sont constitués les territoires du nautisme, définis comme des "espaces concentrant ou reliant en réseaux un certain nombre de lieux et d'attributs nautiques, qu'ils soient matériels ou immatériels, composant une image globale fortement associée au nautisme» (p. 112). Le chapitre sur les aménagements est particulièrement intéressant, notamment lorsque 
Nicolas Bernard développe la notion de stade nautique, ce dernier étant rempli non pas physiquement mais virtuellement par des spectateurs suivant l'évolution de la course sur internet. Le rôle primordial du nautisme dans le processus de recomposition territoriale est également valorisé, la plaisance occupant désormais les espaces laissés vacants par la pêche, le commerce ou la Marine nationale.

Un des grands intérêts de cet ouvrage est d'analyser le nautisme dans ses diverses composantes, économiques certes mais également symboliques. Si le nautisme participe au développement local, c'est grâce aux emplois créés dans la filière nautique mais aussi aux grands événements attirant un nombreux public. Le nautisme, affirme Nicolas Bernard, est indéniablement un marqueur territorial: ainsi, le skipper embarqué à la course au large est l'ambassadeur d'une ville ou d'un département, et l'événement nautique - course au large ou rassemblement de vieux gréements participe à l'élaboration des identités maritimes. S'il dresse un tableau positif du nautisme en France, Nicolas Bernard n'en oublie pas pour autant les points sombres, notamment le très faible nombre de pratiquants comparé à d'autres activités de pleine nature - seuls $1,2 \%$ des séjours touristiques sur le littoral donnent lieu à une activité nautique -, le désengagement des collectivités dans les classes de mer ou encore l'absence de politique commune conduisant à l'éparpillement des initiatives locales.

On peut regretter quelques longueurs - tout le développement sur la place du nautisme dans les recherches scientifiques aurait pu être présenté plus rapidement. Par ailleurs, les hommes sont peu étudiés et la parole de ceux qui pratiquent le nautisme est absente. Mais le propos de l'auteur était avant tout d'« apporter un éclairage utile à l'analyse des nouvelles territorialités »... (p. 296) et cet objectif est indéniablement atteint.

\section{AUTEUR}

\section{ANNE GAUGUE}

Université Blaise-Pascal 